\title{
DINÂMICA DA EMERGÊNCIA DE Parachironomus supparilis EDWARDS, 1931 (DIPTERA, CHIRONOMIDAE) DA FITOFAUNA DE Cabomba piauhyensis GARDNEY, 1844
}

\author{
Kathia C. Sonoda, Susana Trivinho-Strixino \& Giovanni Strixino
}

Abstract

Emergence dynamic of Parachironomus supparilis Edwards, 1931 (Diptera, Chironomidae) from phytofauna of Cabomba piauhyensis Gardney, 1844 - Parachironomus supparilis is a neotropical species with large occurrence in all South America. Although the genus has received detailed taxonomic review, scarce have been the works with an ecological aim, mainly those referring to the numeric dynamic and to emergence patterns. The aim of this paper is to analyze the phenologic dynamic of $P$. supparilis, an important species of the phytophilous fauna of different aquatic macrophytes of lentic environments. A monthly sampling program of Cabomba piauhyensis (Nymphaeaceae) was established from an ox-bow lake of the Mogi-Guaçu River (Infernão Lake), situated at Jataí Ecological Station (Luiz Antônio, SP). The same volume of the macrophyte were collected monthly and placed in a plastic tray and the emergent adults were caught daily, during 30 days in the laboratory for thirty days to collect emergent adults. P. supparilis contributed with $22.6 \%$ of total Chironomidae collected in the period from Apr/96 to Jun/97. With an average numeric density of 39 male adults $/ 10 \mathrm{~g}$ dry weight of $C$. piauhyensis, and with major emergence in the periods of low temperature and precipitation, the species

Programa de Pós-graduação em Ecologia e Recursos Naturais, Laboratório de Entomologia Aquática, Departamento de Hidrobiologia, Universidade Federal de São Carlos. Caixa Postal 676. CEP 13565-905. Rodovia Washington Luís, Km 235, São Carlos, SP, Brasil. E-mail: :strixino@power.ufscar.br 
showed greater intensity of emergence in the months of Aug/96 (84 ind./ $10 \mathrm{~g}$ ) and Jun/97 (75 ind./10g). The presence of adults during the whole period of study seems to indicate that the species is multivoltine, with continuous reproduction.

Key words: Phenologic dynamic, Nymphaeaceae, Parachironomus, Infernão Lake

\section{Resumo}

Parachironomus supparilis é uma espécie neotropical de larga ocorrência em toda a América do Sul. Embora o gênero tenha recebido detalhada revisão taxonômica, escassos têm sido os trabalhos de cunho ecológico, principalmente aqueles referentes à dinâmica numérica e aos padrões de emergência. Com o objetivo de analisar a dinâmica fenológica dessa espécie, característica da fauna fitófila de diferentes macrófitas aquáticas de ambientes lênticos, foi estabelecido um programa mensal de coletas de Cabomba piauhyensis (Sonoda, 1999) (Nymphaeaceae) em uma lagoa marginal (Lagoa do Infernão) do Rio Mogi-Guaçu, localizada na Estação Ecológica de Jataí (Luiz Antônio, SP). Volumes pré-fixados da macrófita foram coletados mensalmente e mantidos em laboratório, onde diariamente durante o período de um mês, foram retirados os adultos de Chironomidae, com auxílio de aspirador entomológico. P. supparilis contribuiu com $22,6 \%$ do total de Chironomidae coletados no período de abril/96 a junho/97. Com densidade numérica média de 39 adultos machos/ $10 \mathrm{~g}$ peso seco de $C$. piauhyensis, e com maior intensidade de emergência nos períodos de baixas temperaturas e menor pluviosidade, a espécie apresentou valores máximos de emergência nos meses de ago/96 (84 ind. $/ 10 \mathrm{~g} \mathrm{p.} \mathrm{s.)} \mathrm{e} \mathrm{de} \mathrm{jun} / 97$ (75 ind./10g p. s.). A presença de adultos durante todo o período de estudo parece indicar que a espécie é multivoltina, apresentando reprodução contínua.

Palavras-chave: Dinâmica fenológica, Nymphaeaceae, Parachironomus, Lagoa do Infernão 


\section{Introdução}

O conhecimento da ecologia de Chironomidae na Região Neotropical baseia-se, principalmente nos estágios imaturos, quase sempre analisados em nível genérico. Ao contrário, a maioria das espécies registradas na região foram analisadas principalmente através da morfologia de suas formas adultas. Dentro desse contexto, são escassas as informações a respeito da ecologia de adultos do gênero Parachironomus Lenz, 1921, cujas larvas são comumente associadas a diferentes macrófitas aquáticas.

Como parte de um estudo sobre a fenologia de Chironomidae associados à Cabomba piauhyensis, este trabalho analisou a dinâmica numérica da emergência de adultos de Parachironomus supparilis, uma das espécies mais abundantes dessa fitofauna.

\section{Área de Estudo}

O estudo foi conduzido numa lagoa marginal ao Rio Mogi-Guaçu, do tipo "ox-bow lake" (Lagoa do Infernão), localizada na Estação Ecológica de Jataí (Luiz Antônio, SP). Trata-se de uma lagoa de infiltração (Feresin, 1994) que se caracteriza por ser um sistema raso e pequeno, no qual as macrófitas aquáticas se desenvolvem abundantemente e cujo comportamento se modifica sistematicamente de acordo com a fase do período hidrológico (Nogueira, 1989).

Além de Cabomba piauhyensis, abundante na região central da lagoa, são também características do local as macrófitas Ceratophyllum demersum, Utricularia sp., Salvinia auriculata, Scirpus cubensis e Eichhornia azurea.

\section{Material e Métodos}

Volumes pré-fixados de $C$. piauhyensis, de bancos de macrófita escolhidos ao acaso, foram coletados mensalmente no período entre abril de 1996 e junho de 1997. O material, acondicionado em sacos plásticos contendo água do local, foi transportado para o laboratório, permanecendo em bandejas plásticas cobertas por gaiolas de emergência por um período de trinta dias. Os adultos coletados diariamente foram fixados em álcool 
70 \%. A montagem das lâminas foi feita segundo metodologia descrita por Pinder (1989), utilizando-se o Meio de Hoyer (Roback, 1976). A identificação dos adultos de $P$. supparilis foi feita com o auxílio de literatura específica (Paggi, 1977; Paggi, 1979; Wiederholm, 1989; Spies et al., 1994; Spies \& Reiss, 1996).

Os valores de densidade numérica foram expressos em número de indivíduos por $10 \mathrm{~g}$ de peso seco de $C$. piauhyensis $\left(\mathrm{n}^{\circ} \cdot 10^{-1} \mathrm{~g}\right.$ p.s.). Os dados climáticos do período foram obtidos junto à Coordenadoria de Assistência Técnica Integral (CATI) da Secretaria da Agricultura e Abastecimento do município de Luiz Antônio.

\section{Resultados e Discussão}

Do total de 1808 adultos machos de Chironomidae obtidos nas gaiolas de emergência, 22,6\% corresponderam à $P$. supparilis.

Com densidade numérica média de 51 ind. $\times 10^{-1} \mathrm{~g}$ de macrófita, $P$. supparilis apresentou emergência durante todo o período de estudo, com menores valores nos meses de outubro e novembro, quando foram registrados maiores valores de precipitação e temperatura (Fig. 1). Notase, portanto, uma possível relação inversa com as condições climáticas.

Ao contrário do padrão observado em $P$. supparilis, a maioria das espécies das regiões temperadas e polares apresenta a emergência restrita aos meses mais quentes, podendo reduzir ou, na maioria dos casos, estar ausente nos meses frios (Dendy, 1971; Danks \& Oliver, 1972; Smith \& Young, 1973; Paterson \& Cameron, 1982). A ausência de estudos sobre emergência de Chironomidae no Brasil, exceto aquele realizado por Walker (1998) em um rio da região amazônica, porém com identificação dos espécimens em nível de subfamília, torna impossível uma análise mais abrangente sobre a dinâmica fenológica da família.

A presença de adultos de Parachironomus supparilis durante todo o período de estudo é um indicativo de que se trata de uma espécie multivoltina. As flutuações numéricas mensais observadas possivelmente reflitam as respostas da espécie às condições climáticas reinantes no período. 

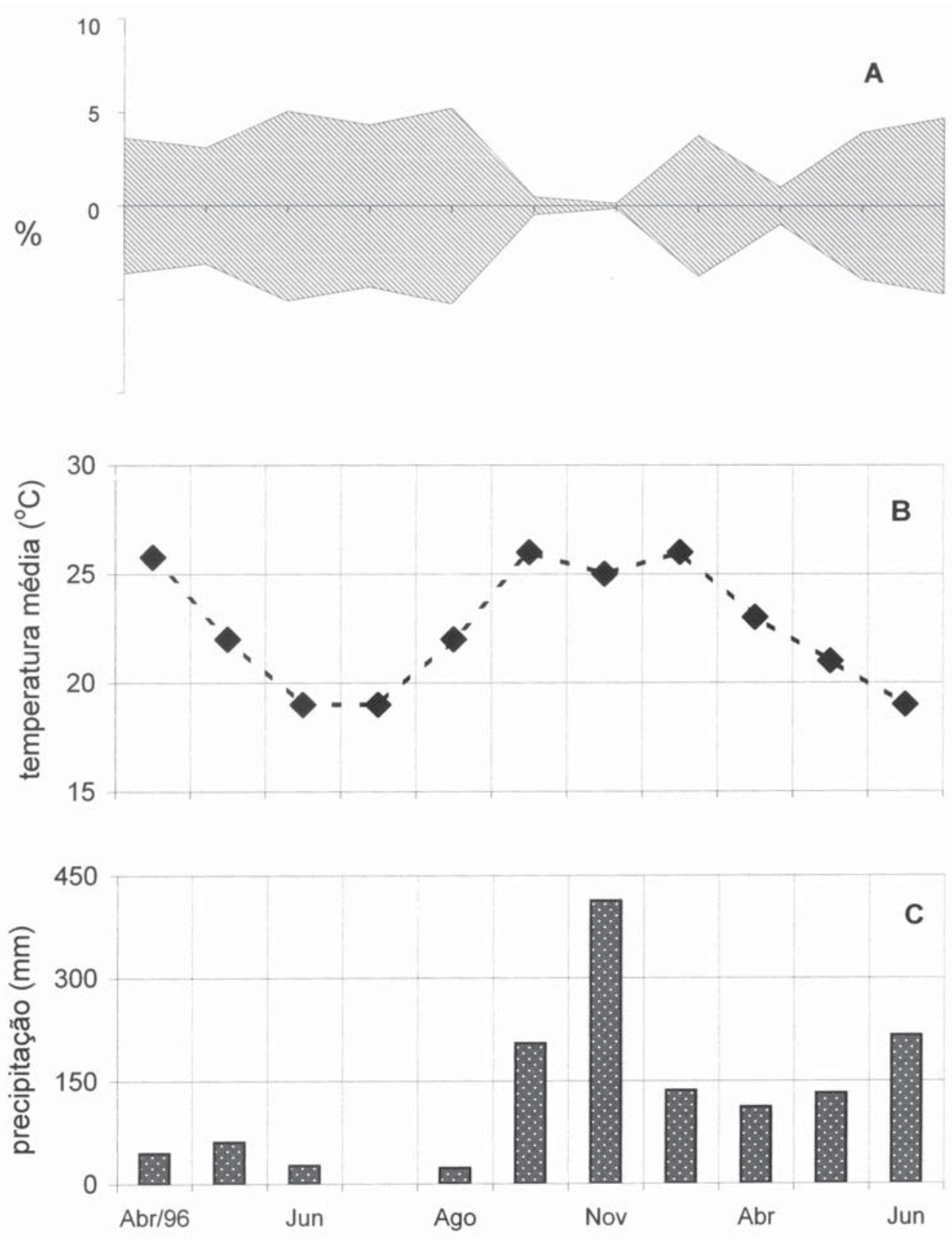

Figura 1: A: Densidade numérica de adultos de Parachironomus supparilis no período de abril/96 a junho/97 na Lagoa do Infernão. B: Temperatura média mensal no mesmo período. C: Índices pluviométricos mensais do período. 


\section{Referências Bibliográficas}

Danks, H.V. \& Oliver, D.R., 1972. Diel periodicities of emergence of some high arctic Chironomidae (Diptera). Can. Entomol. 104:903-916.

Dendy, J.S., 971. Phenology of midges in experimental ponds. Can. Entomol. 103 376-380.

Feresin, E.,G., 1994. Produção de carbono orgânico via fitoplâncton e bacteroplâncton em duas lagoas da planície de inundação do Rio Mogi-Guaçu (Estação Ecológica de Jataí, SP). PPG-ERN/UFSCar, São Carlos, SP. Tese. 108 p.

Nogueira, F.M.B., 1989. Importância das macrófitas aquáticas Eichhornia azurea Kunth e Scirpus cubensis Poepp \& Kunth na ciclagem de nutrientes e nas principais características limnológicas da Lagoa do Infernão (SP). PPG-ERN/UFSCar, São Carlos, SP. Dissertação. 147 p.

Paggi, A.C., 1977. Formas imaginales y preimaginales de quironomidos (Diptera). II. Parachironomus longistilus sp. nov. Limnobios. 1:200-207.

Paggi, A.C., 1979. Dos nuevas especies del genero Parachironomus Lenz (Diptera, Chironomidae) y nuevas citas de quironomidos para la Republica Argentina. Physis - sección B. 38:47-54.

Paterson, C.G. \& Cameron, C.J., 1982. Seasonal dynamics and ecological strategies of the pitcher plant chironomid, Metriocnemus knabi Coq. (Diptera: Chironomidae), in Southeast New Brunswick. Can. J. Zool. 60:3075-3083.

Pinder, L.C.V., 1989. The adult males of Chironomidae (Diptera) of the Holartic region. p. 5 - 9. In: Chironomidae of the Holartic region. Keys and diagnoses. Part 3 - Adult males. Wiederholm, T. (ed.). 532 p.

Roback, S.S., 1976. The immature chironomids of the Eastern United States: I - Introduction and Tanypodinae-Coelotanypodini. Proc. Acad. Nat. Sci. Philad. 127:147-201. 
Smith, V.G.F. \& Young, J.O., 1973. The life histories of some Chironomidae (Diptera) in two ponds on Merseyside, England. Arch. Hydrobiol. 72: 333-355.

Sonoda, K. C., 1999. Chironomidae (Diptera) da fitofauna de Cabomba piauhyensis Gardney, 1844. Dissertação. PPG-ERN. UFSCAR. São Carlos, São Paulo. 96 p.

Spies, M.; Fittkau, E.J. \& Reiss, F., 1994. The adult males of Parachironomus Lenz, 1921, from the Neotropical faunal region. Spixiana, Suppl. 20:61-98.

Spies, M. \& Reiss, F., 1996. Catalog and bibliography of Neotropical and Mexican Chironomidae. Spixiana, Suppl. 22:61-119.

Walker, I., 1998. Population dynamics of Chironomidae (Diptera) in the central amazonian blackwater river Tarumã-Mirim (Amazonas, Brazil). pp. 235-252. In: Ecologia de insetos aquáticos. Série Oecologia Brasiliensis. 5. Nessimian, J. L. \& Carvalho, A. L. E. (eds.). PPGE-UFRJ, Rio de Janeiro, Brasil.

Wiederholm, T. (ed.)., 1989. Chironomidae of the Holartic region. Keys and diagnoses. Part 3 - Adult males. Ent. Scand. Suppl. 34:1-532. 